\title{
Battery Day
}

\section{A CLOSER LOOK AT THE TECHNOLOGY THAT MAKES PORTABLE} ELECTRONICS POSSIBLE

B. esla held its first Battery Day on September 22, 2020 [tesla.com]. What a fantastic world we live in that we can witness the first Apple-like keynote for batteries. Batteries are a part of everyday life; without them, the world would be a much different place. Your cellphone, flashlight, tablet, laptops, drones, cars, and other devices would not be portable and operational without batteries.

At the heart of it, batteries store chemical energy and convert it into electrical energy. The chemical reaction in a battery involves the flow of electrons from one electrode to another. When a battery is discharging, electrons flow from the anode, or negative electrode, to the cathode, or positive electrode. This flow of electrons provides an electric current that can be used to power devices. Electrons have a negative charge; therefore, as the flow of negative electrons moves from one electrode to another, an electrolyte is used to balance the charge by being the route for charge-balancing positive ions to flow.

Let's break this down a bit and uncover the chemical reactions at play within batteries. An electrical current requires a flow of electrons. Where do those electrons come from?

Electrons in the anode are produced by a chemical reaction between the anode and the electrolyte.

Simultaneously, another chemical reaction occurs in the 
cathode, enabling it to accept electrons. These chemical reactions create the flow of electrons, resulting in an electric current.

A chemical reaction that involves the exchange of electrons is known as a reduction-oxidation reaction, or redox reaction.

Reduction refers to a gain of electrons. Thus, half of this reaction-the reduction-occurs at the cathode because it gains electrons. Oxidation refers to a loss of electrons. Therefore, the other half of this reactionoxidation-occurs at the anode because it loses electrons to the cathode. Each of these reactions has a particular electric potential. An electrochemical cell can be made up of any two conducting materials that have reactions with different standard potentials, since the more robust material, which makes up the cathode, will gain electrons from the weaker material, which makes up the anode.

Batteries can be made up of one or more electrochemical cells, each cell consisting of one anode, one cathode, and an electrolyte, as described earlier. The electrodes and electrolyte are generally made up of different types of metals or other chemical compounds. Different materials for the electrodes and electrolyte produce different chemical reactions that affect how the battery works, how much energy it can store, and its voltage.

\section{VOLTS}

The word volt refers to the measure of electric potential. The term derives from the name of the Italian scientist who is credited with inventing the first battery, Alessandro Volta. In 1780, Luigi Galvani, another Italian scientist, 
observed that the legs of frogs hanging on iron or brass hooks would twitch when touched with a probe of some other type of metal. Galvani believed that this was caused by electricity from within the frogs' tissues. He called it "animal electricity."

Volta believed the electric current came from the two different metal types: the hooks on which the frogs were hanging and the probe's different metal. He thought the current was merely being transmitted through, not from, the frogs' tissues. Volta experimented with stacks of silver and zinc layers interspersed with layers of cloth or paper soaked in saltwater and found an electric current flowed through a wire applied to both ends of the pile. Volta also found that the amount of voltage could be increased by using different metals in the pile, leading to what we know today as the scientific unit of a volt [science.org.au].

There are two ways to increase a battery's voltage: stack several cells together or increase a cell's electrochemical potential by choosing different materials.

Combining cells in a series has an additive effect on the battery's voltage. Essentially, the force at which the electrons move through the battery can be seen as the total force as they move from the first cell's anode through all the cells the battery contains to the last cell's cathode.

In contrast, combining cells in parallel increases the battery's possible current, which is defined as the total number of electrons flowing through the cells, but not its voltage.

\section{MEASURING ELECTRICITY}

When you buy a light bulb, the box indicates the wattage 
for the bulb. The watt is a measurement of power. It describes the rate at which electricity is being used at a specific moment. Therefore, a 60-watt light bulb uses 60 watts of electricity at any moment while turned on.

A watt-hour (Wh), on the other hand, is a measurement of energy. It describes the total amount of electricity used over time. You can derive from its name that watt-hours are a combination of watts, the rate electricity is used; and hours, the length of time used. Going back to the example, a 60 -watt light bulb that draws 60 watts of electricity at any moment while turned on, uses 60 watt-hours of electricity in one hour.

Watt-hours will get you only so far, however. To measure the electricity used by a large appliance or a household, the more common term is kilowatt-hour (kWh). A kilowatt is equal to 1,000 watts; therefore, one kilowatt-hour is equal to 1,000 watt-hours.

To measure the output of a power plant or the amount of electricity used by an entire city, megawatt is the more practical term. A megawatt is 1,000 kilowatts or 1 million watts. Going even larger, a gigawatt is 1,000 megawatts, 1 million kilowatts, or 1 billion watts. Gigawatt is the namesake of Tesla's Gigafactory. In 2018 battery production at the Gigafactory in Nevada reached 20 gigawatt-hours [GWh] per year [tesla.com].

\section{ALKALINE BATTERIES}

Most people are familiar with alkaline batteries. These are the batteries you typically use to power toys, electronics, flashlights, etc. The bulk of alkaline batteries produced are single-use, although there are some 
rechargeable alkaline batteries.

Alkaline batteries use zinc as the anode and manganese dioxide as the cathode. The name comes from the alkaline solution used as the electrolyte. The electrolyte is typically potassium hydroxide, which can contain a large number of dissolved ions. The more ions the electrolyte solution can absorb, the longer the redox reaction that drives the battery can keep going.

The zinc anode is usually in powdered form. Powder has a greater surface area for a reaction, which means the cell can quickly release its power. The zinc anode gives up its electrons to the manganese-dioxide cathode, to which carbon, in the form of graphite, is added to improve its conductivity and help it keep its shape.

Alkaline batteries are popular because they have a low self-discharge rate, giving them a long shelf life, and they don't contain toxic heavy metals such as lead or cadmium. They account for the bulk of batteries that are made today, although their place at the top will likely soon be challenged by the lithium-ion batteries in our phones, laptops, cars, and an increasing number of other gadgets.

\section{LITHIUM-ION BATTERIES}

Lithium-ion batteries are popular because of their energy density. Because the energy is dense, your phone can last all day and still be the small, portable, handheld device we are all familiar with. As you likely know from the behavior of your phone, lithium-ion batteries are rechargeable. The name of the battery comes from the fact that lithium ions are involved in the chemical reactions that make up the battery.

In a lithium-ion cell, both electrodes-anode and 


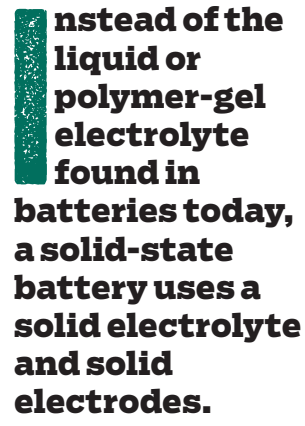

nstead of the liquid or polymer-gel electrolyte found in batteries today, a solid-state battery uses a solid electrolyte and solid electrodes. cathode-are made of materials that can absorb lithium ions. The absorbing action is known as intercalation, which allows the charged ions of an element to be stored inside a material without significantly disturbing it. The lithium ions are paired with an electron within the structure of the anode. When the battery discharges, the intercalated lithium ions are released from the anode and travel through the electrolyte solution to be intercalated in the cathode.

A lithium-ion battery starts its life in a state of full discharge: All its lithium ions are intercalated within the cathode, and its chemistry cannot yet produce any electricity. Before the battery can be used, it needs to be charged. As the battery is charged, an oxidation reaction occurs at the cathode, meaning that it loses some negatively charged electrons. An equal number of positively charged intercalated lithium ions are dissolved into the electrolyte solution to maintain the charge balance in the cathode. These travel over to the anode, where they are intercalated, or absorbed, into what is typically graphite. This intercalation reaction also deposits electrons into the graphite anode, to pair with the lithium ions.

There are many other types of batteries, but for the context of this article you primarily need to understand lithium-ion batteries. (Some people might, however, be interested in a urine-powered battery [newatlas.com].)

\section{NEW TECHNOLOGIES}

\section{Solid-state batteries}

Instead of the liquid or polymer-gel electrolyte found in batteries today, a solid-state battery uses a solid electrolyte and solid electrodes. Recall that positive ions 
flow through the electrolyte to balance the electrons' negative charge. Today, batteries are quite efficient at transferring positive ions since a liquid electrolyte is in contact with the electrodes' entire surface area. Using a solid makes this a bit harder. Imagine the difference between dipping a chip in soup and dipping it into chopped tomatoes. The soup would cover more of the chip's surface area than would the chopped tomatoes.

Why use a solid electrolyte if it is less efficient? Today's lithium-ion batteries typically rely on flammable liquids as the electrolyte. By using a solid electrolyte, batteries can be less prone to bursting into flame. You may remember Samsung's Galaxy Note 7, which had the unfortunate side effect of catching fire [time.com]. Solid electrolytes provide a much safer alternative.

Solid electrolytes typically tend to be either solid polymers at high temperatures or ceramics at room temperature. The downside of solid polymers is they need to operate at temperatures above $220^{\circ} \mathrm{F}\left(105^{\circ} \mathrm{C}\right]$ [qz.com]. That is certainly not practical for a handheld device such as a phone or tablet but could work for storing energy to power a home.

A few companies are working on using ceramics at room temperature to create a solid-state battery. Toyota has been talking about its battery for years [cen.acs. org] and aims to have it completed in 2025 [caranddriver. com]. Startups such as Solid Power [solidpowerbattery. com] and A123 Systems (with the help of Ionic Materials [ionicmaterials.com]] aim to do the same.

A lot of the novel research being done on solid-state batteries is the work of Jürgen Janek [iop.org]. Janek 
recently published a benchmark of the performance of all-solid-state lithium batteries [nature.com]. Another high-profile battery scientist, Gerbrand Ceder, published a paper on interface stability in solid-state batteries [nature.com]. New research on solid-state batteries is being published quite frequently. Although there are many skeptics of solid-state batteries, since they have yet to be commercially delivered and scaled, I would not dismiss it from having a seat at the table in the future.

Nuclear batteries

So far we have discussed only batteries powered by chemical reactions, such as those in flashlights, phones, and other gadgets. Chemical batteries, also known as galvanic cells, discharge in a given amount of time and need to be either thrown away or recharged. Is there a type of battery that could last long term?

Nuclear batteries, or atomic batteries, use the energy of beta decay and are known as betavoltaics. They are receiving research attention because they could last much longer than those powered by chemical reactions. Radioactive isotopes used in nuclear batteries have halflives ranging from tens to hundreds of years, so their power output remains nearly constant for a very long time.

If nuclear batteries can last from tens to hundreds of years, why are they not being used everywhere today? Doesn't everyone want a phone that could last at least a decade without needing to be charged?

Nuclear batteries have a few side effects. They cannot be turned off; electrons are continually being produced, even when they are not needed. Research is being done 
into stimulating beta decay [nist.gov], which would create more current on demand, allowing the output to drop to almost nothing when it is turned off.

Another downside is the power density of betavoltaic cells is much lower than that of chemical batteries. It is interesting to note that betavoltaics were used in the 1970s to power cardiac pacemakers, before being replaced by cheaper lithium-ion batteries, even though the lithiumion battery has a shorter lifetime.

In 2016 Russian researchers from the National University of Science and Technology MISiS presented a prototype betavoltaic battery based on nickel-63 [phys. org]. A downside of using nickel-63 is that it is not readily available, making their research hard to commercialize. City Labs sells a betavoltaic battery with a 14.4-year half-life, which you can buy today starting at $\$ 1,000$ [citylabs.net], but you would need 1.2 million of these just to have one watt of power. NDB [ndb.technology] is a startup working on a nano diamond battery that could last for thousands of years [techcrunch.com]. UPower Technologies [upowertech.com] is another startup working on a megawatt-scale atomic generator.

\section{Silicon anode}

Today the material typically used for the anode is graphite because it is economical, reliable, and relatively energy dense, especially compared with current cathode materials. The limiting factor of lithium-ion batteries is the amount of lithium that can be stored in the electrodes. Using silicon as the material for the anode, rather than graphite, allows around nine times more lithium ions to be 
held in the anode.

The ability to store more lithium ions using silicon sounds amazing; why isn't everyone doing this? The problem is a silicon anode swells to three to four times its original volume when it absorbs lithium ions. Making the casing bigger doesn't circumvent the problem because the expansion causes the silicon to fracture, leading to failure of the battery. A passivation layer, also known as the SEl (solid electrolyte interphase), forms on electrode surfaces from the decomposition of electrolytes. This passivation layer typically inhibits further electrolyte decomposition, giving the battery a longer life. The absorption of lithium ions gums up this layer making it less effective.

"With silicon, the cookie crumbles and gets gooey." -Elon Musk.

As a solution to this problem, many companies use silicon as a fraction of the anode material, but such materials are expensive and highly engineered. Examples include silicon structured in $\mathrm{SiO}$ [silicon dioxide] glass [\$6.60 per kWh], silicon structured in graphite [ $\$ 10.20$ per kWh], and silicon nanowires [ $\$ 100$ per kWh] [youtube. com]. Sila Nanotechnologies [silanano.com] is using silicon as its anode material. Amprius [amprius.com] claims to use silicon for 100 percent of the anode material with silicon nanowires, a highly engineered, expensive material. Advano [advano.io], Enevate [enevate.com], and Enovix [enovix.com] are startups working on a silicon solution for the anode material. 


\section{TESLA'S BATTERY DAY}

At Tesla's Battery Day event, the company announced many changes to its battery that encompass more than just the materials used. Tesla has on staff the renowned battery scientist Jeff Dahn. His most recent papers, "A Wide Range of Testing Results on an Excellent Lithium-Ion Cell Chemistry to be Used as Benchmarks for New Battery Technologies" [iop.org] and "Is Cobalt Needed in Ni-rich Positive Electrode Materials for Lithium-Ion Batteries?" [iop.org], help give some insight into what Tesla has been working on.

The Battery Day outcomes could increase Tesla vehicles' range while being more economical; the plan is to halve the cost per kilowatt-hour. Most startups lexcept for Sila Nanotechnologies, which seems to be most closely aligned with Tesla's methodology [silanano.com]] in this space tend to take a single design decision into account for their products-for example, anode material-and focus on that. Tesla, on the other hand, took a broader approach. It took into account not only the materials for the cathode and anode, but also the cell design, factory, and integration with the vehicle, illustrated in figure 1. (Tesla claimed in its presentation there were more aspects not mentioned that it could improve in the future.]

Let's break down each of these improvements.

\section{Cell design}

For Tesla's batteries, when discharging, the positive ions flow over the tabs, while the lithium ions flow from the anode to the cathode, as shown in figure 2. The tabs allow a cell's energy to be transferred to an external source. 


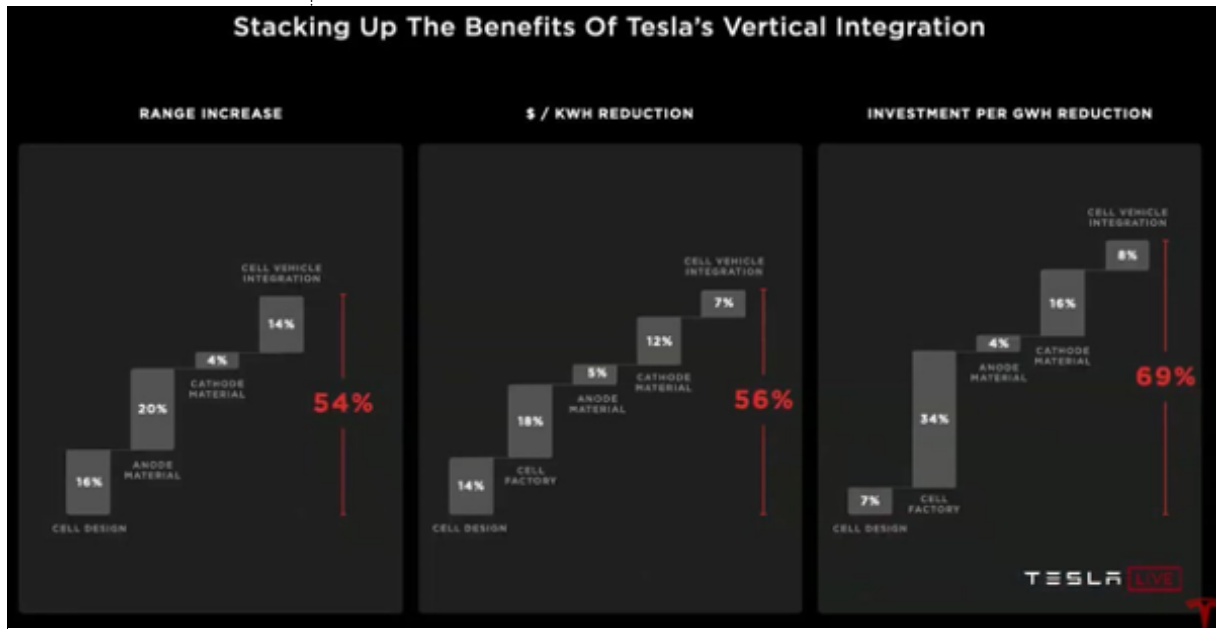

Source: Tesla's Battery Day Presentation https://www.youtube.com/watch?v=l6T9xleZTds

\section{FIGURE 2: BATTERY DISCHARGE}

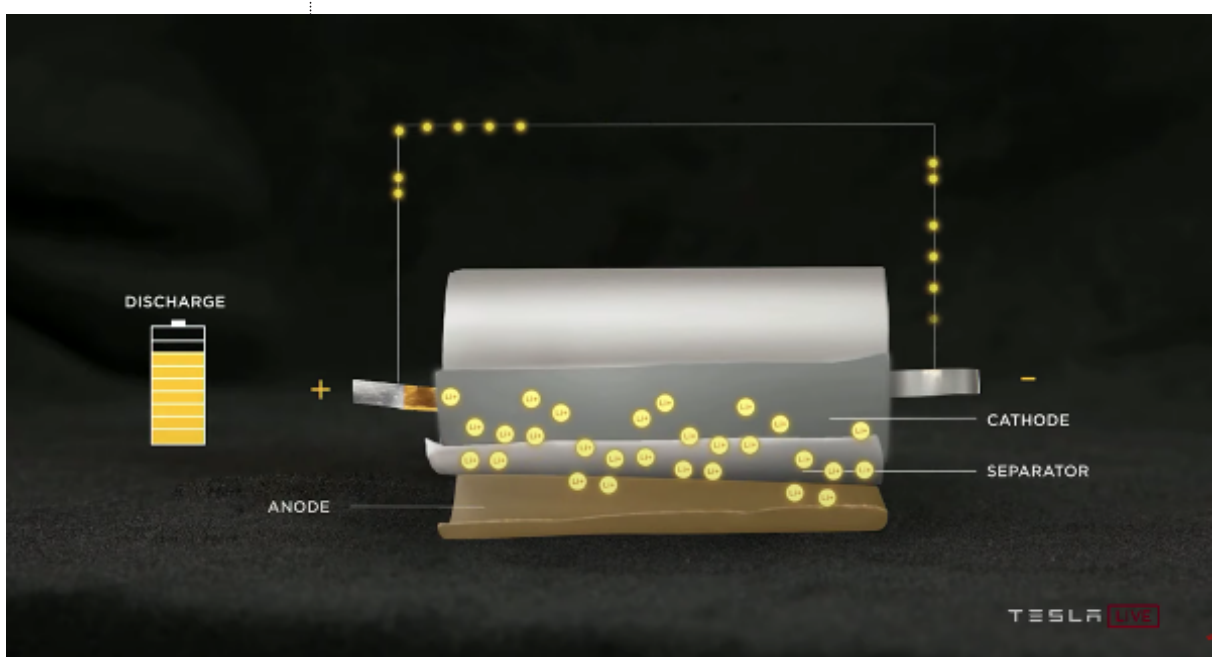

Source: Tesla's Battery Day Presentation https:I/www.youtube.com/watch?v=l6T9xleZTds 
The Tesla team sought to increase the cell size to 46 millimeters, which optimizes vehicle range and cost reduction. Increasing the cell's size, however, has a negative side effect on supercharging because of thermal issues. To circumvent these issues, the Tesla team removed the tabs, calling the new design "tabless."

The tabless design leads to simpler manufacturing, fewer parts, and a fivefold reduction of the electrical path. Going from a 250-millimeter to a 50-millimeter electrical path length leads to substantial thermal benefits. The length of the electrical path is significant because the distance the electron has to travel is much less. Even though the cell is much bigger, the power-to-weight ratio is better than a smaller cell with tabs.

Why does this new tabless design matter? Instead of calling it tabless, Tesla could have called it many tabs, because each of the folded pins is a tab, as shown in figure 3. What is the function of a tab?

Consider this analogy: When I was growing up, my family would always leave sporting events before they ended to avoid the crowd trying to exit the stadium. If we had stayed to the end of the event, the exiting process would have taken more time and would be uncomfortable since everyone would be trying to leave through a few exits at the same time. They get closer and closer to one another, and the environment becomes hot and rowdy. If you think of the exiting people as electrons, a stadium with a single exit is similar to a battery's behavior with a single tab; electrons are all trying to leave through the single tab and bumping up against one another until they heat up.

Tesla's new design has multiple tabs, equivalent to a 


\section{FIGURE 3: TABLESS CELL}

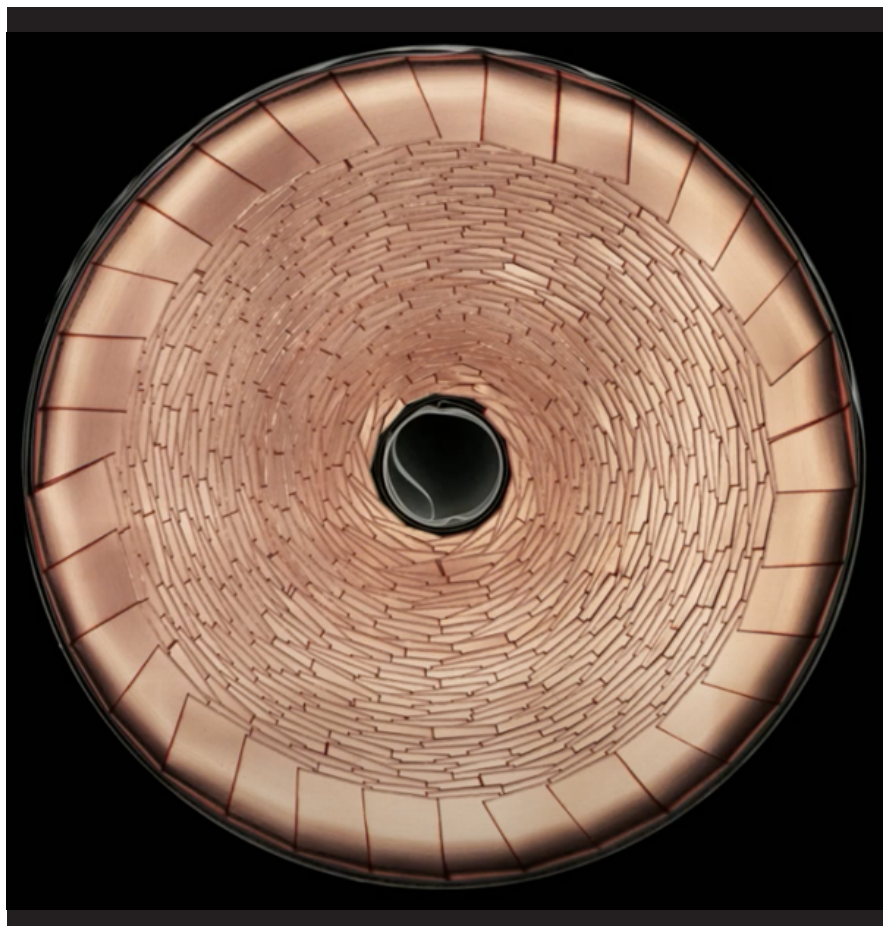

Source: Tesla's Battery Day Presentation https://www.youtube.com/ watch? $v=16 T 9 \times 1$ leZTds

stadium with lots of exits. Now people, or electrons, can exit quickly while staying cool and calm.

Tesla didn't provide many details in its presentation on the new tabless design and its implementation, but it can be attributed to "secret sauce."

Manufacturing a cell consists of an electrode process where the active materials are coated into films onto foils; the coated foils are then wound in the winding process. 
The roll is assembled into the can, sealed, and filled with electrolyte and then sent to formation, where the cell is charged for the first time. Recall that a lithium-ion battery starts its life in a discharged state. For a battery cell with tabs, manufacturing is much more complicated. When the cell with tabs is going through the assembly line, it has to keep stopping where all the tabs are so you can't do continuous motion production. It is also a lot more error prone.

"It is really a huge pain in the ass to have tabs from a production standpoint." -Elon Musk.

The new batteries are 46 millimeters by 80 millimeters, leading to the name 4680 , referring to the diameter and length. Previously, an extra zero was added onto the end of the name but was removed since it had no purpose.

The 4680 batteries have five times more energy with six times the power and enable a 16 percent range increase. At the battery-pack level, the form-factor improvements alone result in a 14 percent reduction in cost per $\mathrm{kWh}$.

\section{Cell factory}

Earlier we learned a bit about how removing tabs from the battery cells simplifies the manufacturing process. In an assembly line, you don't want the line to stop and start, but continuously move. Stopping the process leads to inefficiency. The Tesla team aims to speed up its process to make one factory be multiple scales of efficiency better than a typical battery factory.

We also learned that the electrode process is where 
the active materials are coated into films onto foils. The wet step of the electrode process begins with mixing, which occurs when the powders are mixed with either water or a solvent, typically a solvent for the cathode. The mix then goes into a large coat-and-dry oven, tens of meters long, where the slurry is coated onto the foil and dried. The solvent then has to be recovered. Finally, the coated foil is compressed to the final density. This process is complex and inefficient, especially since humans need to transport the mix from the mixing step to the ovens. It is also inefficient because of the need to put the solvent in and then recover it.

One significant change Tesla is making is skipping the solvent step of the electrode coating's wet process in favor of a dry process, which transforms the powder directly into film. This technology stemmed from Tesla's acquisition of Maxwell at the beginning of 2019 [electrek.co]. On Battery Day, Musk mentioned that since the acquisition, Tesla is now on the fourth revision of the equipment that turns powder into film. He noted, "There is still a lot of work to do. There is a clear path to success but a ton of work between here and there." When this process is scaled up, it results in a tenfold reduction in footprint and an equal reduction in energy, as well as a massive decrease in CapEx (capital expenditure).

The manufacturing step known as formation is where the cell is charged for the first time and its quality is verified. Formation is typically 25 percent of the CapEx investment. The Tesla team improved density and cost effectiveness by using their knowledge from cars and power-wall charging and discharging. This led to an 
86 percent reduction in CapEx investment per GWh in formation and a 75 percent reduction in footprint. For a factory that previously had an output of $150 \mathrm{GWh}$, this translates to that same factory putting out 1 TWh using the more efficient processes. At the battery-pack level, this led to an 18 percent reduction in cost per $\mathrm{kWh}$.

\section{Anode material}

Tesla announced it was moving to silicon as its anode material. Silicon is the most abundant element in the earth's crust after oxygen. Rather than creating a highly engineered material that would be expensive, Tesla will use raw silicon and design for it to expand. The silicon's surface will be stabilized through an elastic, ion-conducting polymer coating and a highly elastic binder and electrolyte.

Tesla's silicon costs $\$ 1.20$ per $\mathrm{kWh}$, whereas the other solutions covered here cost anywhere from $\$ 6$ per $\mathrm{kWh}$ to upwards of $\$ 100$. Using silicon will lead to a 5 percent reduction in cost per $\mathrm{kWh}$ at the battery-pack level and a 20 percent longer range for Tesla vehicles.

\section{Cathode material}

A helpful analogy for understanding the cathode is to think of it as a bookshelf, as illustrated in figure 4 . In this case, the lithium ions are books. The most efficient bookshelf holds the most books while still being stable enough to retain its structure as the books are taken and returned.

The Tesla team aims to increase the amount of nickel in its cathode material since it is less expensive and has the highest energy density [as shown previously]. Cobalt is typically used as a cathode material because it is very 


\section{FIGURE 4: BATTERY CATHODE}

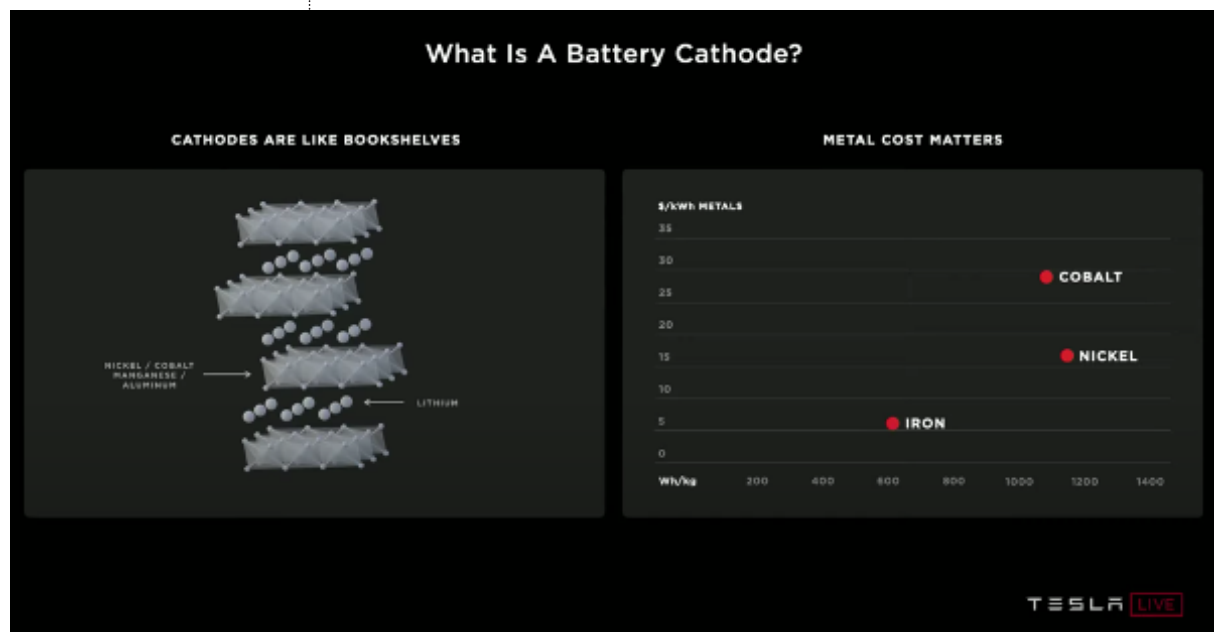

Source: Tesla's Battery Day Presentation https://www.youtube.com/watch?v=l6T9xleZTds

stable. The Tesla team, however, aims to leverage novel coatings and dopants to stabilize nickel better and remove cobalt entirely from its materials. Removing cobalt will lead to a 15 percent reduction in the cathode's cost per kWh.

The cost and availability of materials were important considerations. Availability was not an issue for the anode material since silicon is abundant. The same goes for lithium, which is also highly accessible. For nickel, on the other hand, the Tesla team is diversifying the amount it is using for each type of vehicle.

The team also simplified the cathode-manufacturing process by removing all the legacy parts. According to the Battery Day presentation, the cathode manufacturing process, which is 35 percent of the cathode cost per $\mathrm{kWh}$, 
had not had a fresh look in a long time and was wildly inefficient.

"If you take a look at the 'It's a small world journey' of I am a nickel atom, and what happens to me, it's crazy, you're going around the world three times, there is a moral equivalent of digging the ditch, filling in the ditch, and digging the ditch again. It's total madness." -Elon Musk

A typical cathode process starts with the metal from the mine being turned into an intermediate material called metal sulfate, which, in turn, is processed again. The Tesla team removed the intermediate step of turning the metal into metal sulfate along with a bunch of other unnecessary steps. It also localized the cathode materials to the U.S., which decreased the number of miles required for the materials to travel. This led to a 66 percent reduction in CapEx investment, a 76 percent reduction in process cost, and zero wastewater. The cathode material improvements led to a 12 percent reduction in cost per $\mathrm{kWh}$ at the battery-pack level.

Cell vehicle integration

In the early days of aircraft, fuel was carried as cargo. Later, fuel tanks were made in the shape of the wings. This was a breakthrough because the wings, which are critical to the airplane's function, now could be used for another purpose. The fuel tank was no longer cargo but fundamental to the structure of the aircraft. Tesla intends to do the same for cars. 
Removing the intermediate structure in the battery pack allows the cells to be packed more densely. Instead of having supports and stabilizers in the battery cells, making up the intermediate structural elements, the battery pack itself is structural. Tesla had been filling the battery packs with a flame retardant. The new battery packs are filled with a flame retardant and structural adhesive, giving them stiffness and stability without intermediate structural elements. This makes the structure even stiffer than a regular car.

The cells can now be moved more toward the center of the vehicle because the volumetric efficiency is better, avoiding the risk of a side impact potentially contacting the cells. This also allows the car to maneuver better because the polar moment of inertia is improved-much the way an ice skater can turn better with arms close to the body rather than extended out.

The improvements to the battery-pack integration led to a 10 percent mass reduction in the car's body, a 14 percent range increase, and 370 fewer parts. The smaller, integrated battery and body also help increase the efficiency of manufacturing. This has resulted in a 55 percent reduction in CapEx investment and a 35 percent reduction in floor space. At the battery-pack level, the integration improvements mean a 7 percent reduction in cost per kWh.

The sum of all these improvements-including cell design, factory, materials, and vehicle integrationachieves the goal of halving the cost per $\mathrm{kWh}$. Producing more affordable electric vehicles broadens Tesla's market to include new buyers, thereby reducing the number of 
gas-powered vehicles on the road.

\section{SUMMARY}

It is satisfying to see a technology we all rely on day to day get its time in the spotlight. Although not mentioned during Battery Day, if Tesla were to achieve 400 watt-hours per kilogram, a zero-emissions jet just might be on the horizon. Now that batteries are vertically integrated into Tesla's product, you can only imagine that the software will track more data on battery efficiency, leading to more improvements in the future.

It is encouraging to see Tesla take a fresh look at making more efficient and cost-effective batteries. The level of thought and detail put into rethinking old processes to make them more efficient is inspiring. The Tesla team didn't just look at one angle but all the angles: cell design, manufacturing, vehicle integration, and materials. There is a clear why for every decision made that boils down to economics, not just technical gains. Hopefully, we will see another core technology in the spotlight soon.

Jessie Frazelle is the cofounder and chief product officer of the Oxide Computer Company. Before that, she worked on various parts of Linux, including containers, as well as the Go programming language.

Copyright (C) 2020 held by ownerlauthor. Publication rights licensed to ACM. 